\title{
Therapie mit Chondroitinsulfat: Die Reinheit ist entscheidend
}

\author{
Chondroitinsulfat ist eine etablierte Substanz in der Behandlung der Osteoarthritis. Allerdings zeigen Studien, dass \\ die Wirkung von Chondroitinsulfat eng mit Qualität und Reinheit korreliert.
}

\begin{abstract}
Literatur:
1. Dorner TE, Stein KV (2013) Prevalence and status quo of osteoarthritis in Austria. Analysis of epidemiological and social determinants of health in a representative cross-sectional survey. Wien Med Wochenschr 163:206-211 2. Hochberg M, Chevalier $X$, Henrotin $Y$ et al (2013) Symptom and structure modification in osteoarthritis with pharmaceutical-grade chondroitin sulfate: what's the evidence? Curr Med Res Opin 29:259-267

3. Kullich W, Leeb B, Mur E (2014) Therapie der Arthrose mit Chondroitinsulfat. J Miner Stoffwechs 21(Suppl 1):1-10

4. ArnoldW, Fullerton DS, Holder S et al (2007) Viscosupplementation: managed care issues for osteoarthritis of the knee. J Manag Care Pharm 13(4 Suppl):3-19, quiz 20-22 5. Jordan KM, Arden NK, ältere Menschen betroffen. Einer Studie zu Prävalenz und Status quo der Osteoarthritis in Österreich aus dem Jahr 2013 zufolge leiden etwa $12 \%$ der Männer und 19\% der Frauen an Arthrose [1]. In Zukunft ist, aufgrund der demographischen Entwicklung, eine Zunahme der Betroffenen zu erwarten.

Am häufigsten von einer Arthrose in Mitleidenschaft gezogen ist das Knie (30-35\% aller Arthrosen), gefolgt von Hand- und Fingergelenken (30-35\%) und der Hüfte (10-12\%). Derzeit verfügbare medikamentöse Therapie-Optionen sind meist nur symptomatisch wirksam. Eine Substanz, die seit längerer Zeit bei der Behandlung von Arthrosen zum Einsatz kommt, ist Chondroitinsulfat (CS). Vorhandene Daten deuten auf eine signifikante symptomatische Wirkung von Chondroitinsulfat bei verschiedenen Arthroseindikationen hin [2].
\end{abstract} Doherty M et al (2003) Standing Committee for International Clinical Studies Including Therapeutic Trials ESCISIT. EULAR Recommendations 2003: an evidence based approach to the management of knee osteoarthritis: report of a task force of the Standing Committee for International Clinical Studies Including Therapeutic Trials (ESCISIT). Ann Rheum Dis 62:1145-1155 6. Tat SK, Pelletier JP, ineau F et al (2010) Variable effects of 3 different chondroitin sulfate compounds on human osteoarthritic cartilage/chondrocytes: relevance of purity and production process. J Rheumatol 37:656-664
Von einer Arthrose sind vor allem

\section{Auf- und Abbau im Ungleich- gewicht}

Die Proteoglykane des Knorpels unterliegen einem laufenden Verschleiß und müssen daher von den Chondrozyten nachgebildet werden. Die mittlere Halbwertszeit von Proteoglykanen im Knie beträgt rund 400 Tage, in der Hüfte etwa 800 Tage [3].

In gesunden Gelenken sind Matrixab- und Matrixaufbau im Gleichgewicht. Wird weniger Matrix synthetisiert als abgebaut, beginnen arthrotische Prozesse. Zu den Risikofaktoren für das Entstehen einer Arthrose zählen: Übergewicht, Gelenkfehlstellungen, genetische Prädisposition, Mikrofrakturen im subchondralen Knochen und Traumata. Neben dem Alter können auch metabolische Erkrankungen, Hormontherapien, Entzündungen und Funktionsstörungen des Immunsystems den Abbau des Gelenkknorpels vorantreiben [4].

\section{Knorpelschutz}

Bei der Therapie der Arthrose geht es vor allem um Schmerzreduktion, eine Verbesserung der Gelenksfunktion und eine Verzögerung der Progression und Folgeschäden [5].

Denkbar sind verschiedene Ansatzpunkte für eine chondroprotektive Behandlung: Die Steigerung der Proteoglykan-Syntheserate, das Verbessern der Qualität der synthetisierten Moleküle, das Verringern kataboler Vorgänge - zum Beispiel durch Reduzieren des enzymatischen Knorpelabbaus durch Matrix-Metalloproteinasen (MMP) - und die exogene Zufuhr von Proteoglykan-Bestandteilen wie etwa Chondroitinsulfat.

\section{Qualitätsmerkmal Reinheit}

Chondroitinsulfat zählt zu den gut etablierten, oral verabreichten „symptomatic slow-acting drugs" für Osteoarthritis (SYSADOA). Dabei belegen Studien, dass die Qualität von Chondroitinsulfat in zahlreichen Nahrungsergänzungsmitteln sehr stark schwankt und dass Nahrungsergänzungsmittel mehr oder weniger große (und ex lege nicht nachprüfbare) Mengen an Chondroitin- sulfat enthalten können. Vor allem im Hinblick auf unspezifische Effekte sollte der Reinheitsgrad aber gut dokumentiert sein [2].

Deshalb empfiehlt sich, Chondroitinsulfat in Arzneimittelqualität einzusetzen. Versuche konnten zeigen, dass eine Reinheit von $90 \%$ wesentlich weniger effizient auf katabole Marker wie IL- 6 und MMP-1 war als $96 \%$ Reinheit. Bei $99 \%$ konnte sogar die IL-1-induzierte Hemmung der Kollagen-II-Genexpression aufgehalten werden [6].

Die Grundlagenforschung konnte zeigen, dass von außen zugeführtes Chondroitinsulfat im arthrotischen Gelenk verschiedene Effekte erzielen kann: Chondroitinsulfat kann Chondrozyten, Fibroblasten und Synovialzellen stimulieren, was zu einer erhöhten Proteoglykansynthese führt. Es wirkt chondro- und zellprotektiv. Chondroitinsulfat kann zudem proinflammatorische Faktoren reduzieren, die Apoptose modifizieren und das Gleichgewicht zwischen anabolen und katabolen Prozessen in der Knorpelmatrix verbessern [3].

Quelle:

Unterlagen der Sanova Pharma GesmbH

rheuma plus $2014 \cdot 13: 8$

DOI 10.1007/s12688-014-0008-9

Online publiziert: 13. November 2014

(c) Springer-Verlag Wien 2014 